\title{
Hemostatic Resuscitation
}

\author{
N. R. McMullin, J. B. Holcomb, and J. Sondeen
}

\section{Introduction}

Advances in surgical and critical care medicine frequently parallel the course of armed conflict. Indeed, surgery is a specialty born of warfare and will continue to drive advancements as mankind finds new and more lethal methods of combat. As hemorrhage is far and away the leading cause of potentially survivable death on the battlefield, the methods of resuscitation and blood transfusion continue to evolve. The critical role that blood plays in resuscitation of the critically injured patient was first explored during World War I for the treatment of 'wound shock'. Type $\mathrm{O}$ whole blood was collected in sterile glass bottles containing citrate and transfused into patients prior to surgery. During the years following World War I, blood component fractionation became available, blood banking was initiated, and the transfusion of packed red blood cells (RBCs), fresh frozen plasma (FFP) and platelets became a mainstay of the trauma management paradigm. However, in times of war the variable availability of short-lived platelets, FFP, and cryoprecipitate inevitably leads back to the resurrection of fresh whole blood transfusion. Fresh whole blood, though not without some risk, restores the hemostatic mechanism and provides volume and oxygen-carrying capacity.

Several studies have questioned the universal benefit of RBC transfusion for trauma patients, having found immunosuppression and dilutional coagulopathy in patients who receive massive transfusion. Additionally, standard component therapy dilutes platelets and coagulation factors in these patients. Massive transfusion is further hampered by a pressing need for new intravascular methods of preventing ongoing blood. Fluid for optimal resuscitation should be widely available and have a stable shelf life, and combine the safety of blood components with the efficacy, resuscitative and hemostatic effects of fresh whole blood. Such a fluid would truly provide 'hemostatic resuscitation'. Hemostatic resuscitation as an initial resuscitation regimen would restore the hemostatic mechanism, correct, or more importantly, prevent coagulopathy and would also enhance tissue perfusion. In this chapter, we describe the recent widespread use of fresh whole blood on the battlefield, the controversy surrounding massive transfusion of stored packed RBCs and other blood components as well as promising new developments in the arena of blood component substitutes. 


\section{Fresh Whole Blood}

Combat medicine is an ebb and flow of casualties. Several days may pass when there are no casualties, followed by an onslaught of wounded. Casualties from explosive devices, such as those seen in Operation Iraqi Freedom and civilian terrorist attacks, tend to result in a large number of casualties at one time. This paradigm does not lend itself to component therapy. Packed RBCs, plasma, and crystalloid solutions may be in abundance, but a five-day shelf life prohibits the banking of platelets. The casualties who receive massive transfusions ( $>10$ units packed RBCs) quickly deplete precious stores. FFP bags, not designed to support the temperature $\left(-70^{\circ} \mathrm{F}\right)$ associated with dry ice used during transport, become brittle and break one third of the time during the thawing process. Platelets generated by plasmapheresis became available only two years after the start of the war in Iraq, require significant and dedicated resources and availability remains limited. Limited blood products, manpower shortages, and warming methods increase the vulnerability of wounded soldiers to the onset of a second enemy: hypothermia, acidosis and coagulopathy.

In an attempt to resolve this problem combat surgeons often resort to the 'walking blood bank'. Predetermined donors are mobilized and fresh whole blood is collected, tested, and given to the wounded. Fresh whole blood protocols were followed in post-war Kosovo and in Somalia where over 120 units of fresh whole blood were collected and administered to critically wounded patients $[1,2]$. A combat support hospital deployed to Baghdad transfused 598 units of fresh whole blood over a 6 month period [3]. Reports of the rapid positive response of recipients to fresh whole blood are thought-provoking and suggest profound possibilities for fresh whole blood transfusion. In combat, fresh whole blood for massive transfusion becomes a blood bank multiplier, providing within a single unit, RBCs, volume, coagulation components, and functional platelets in a warm fluid.

In 1999, Pearce and Lyons contrasted blood product usage from World War II and Korea to the practice in Vietnam [4]. In World War II and Korea, resuscitation consisted of colloid in the form of concentrated plasma and fresh whole blood. However, the incidence of hepatitis transmission rose to unacceptable levels - as high as $21 \%$ in some units in Korea [5]. In Vietnam, tested units of packed RBCs and crystalloid solutions (lactated Ringer's) were used and the incidence of infectious disease decreased. However, complications of the switch from fresh whole blood to stored RBCs became evident in Vietnam as acute respiratory distress syndrome (ARDS) or 'Da Nang Lung' was so prevalent that it became the focus of thousands of studies and book chapters. In comparison, very few cases of ARDS were described during World War II and none during Korea despite the administration of large volumes of whole blood and colloid [4].

These observations from the front lines of the advantages of fresh whole blood transfusion have been borne out in the civilian literature. In particular, two well designed, prospective studies support the clinical testimony of combat surgeons that fresh whole blood improves coagulopathy and decreases blood loss when compared to component therapy. The first study randomized cardiopulmonary bypass (CPB) patients to receive either one unit of fresh whole blood or 10 units of platelet concentrates after surgery [6]. The patients who received one unit of fresh whole blood increased platelet counts by $34 \pm 17 \times 10^{9} / 1$, an increase equivalent to four to six units of platelets. Platelet aggregation response to collagen and epinephrine after fresh whole blood transfusion was superior to that achieved by 10 units of platelets. 
Furthermore, bleeding time after administration of one unit of fresh whole blood approximately equaled bleeding time following eight units of platelets. In the second study, the beneficial effects of fresh whole blood on the coagulation cascade were demonstrated in a double-blinded, randomized controlled study comparing the use of fresh whole blood less than 24 hours old $(n=52)$, fresh whole blood between 24 and 48 hours old $(n=57)$, and reconstituted blood using packed RBCs, plasma, and platelets $(n=52)$, in a pediatric population undergoing CPB operations [7]. The 24-hour blood loss was no different between the two whole blood groups. The group that received reconstituted products had an increase in average blood loss that was significantly greater than either of the fresh whole blood groups $(p=0.03)$. After age stratification, the 24-hour blood loss for children less than 2 years old who received reconstituted blood was $85 \%$ greater than those who received fresh whole blood $(p=0.001)$. When platelet aggregation times were compared among the three groups, the group that received the reconstituted blood had a greater incidence of abnormal studies in the presence of the agonists ADP, epinephrine and collagen $(\mathrm{p}<0.001, \mathrm{p}=0.02$, and $\mathrm{p}=0.007$, respectively). Other recent studies have linked increasingly negative outcomes to each unit of packed RBCs received.

Fresh whole blood transfusion has also been shown to improve hemodynamic and oxygen delivery parameters in animal studies. One such study comparing transfusion of packed RBCs with whole blood in a canine model of hemorrhagic shock demonstrated that animals resuscitated with packed RBCs had significantly less hemodynamic recovery, demonstrated by lower mean arterial pressure and cardiac output $(\mathrm{p}<0.05)$ [8]. Resuscitation with packed RBCs elevated total peripheral resistance when compared with resuscitation with fresh whole blood, suggesting vasoconstriction or obstruction of the peripheral vasculature by noncompliant RBCs [8]. This study corroborated findings of an earlier study that showed improved cardiac output and oxygen delivery in dogs resuscitated with whole blood compared to those resuscitated with crystalloid or colloid [9].

In an attempt to explain these observations of improved oxygen delivery, macroaggregated albumin clearance rates from the lungs of hemorrhaged rats were studied. Rats that received fresh whole blood demonstrated improved clearance of the tracer compared to animals resuscitated with either crystalloid or colloid [10]. Another rat model of hemorrhage and resuscitation demonstrated that fresh whole blood for resuscitation provided superior end organ perfusion by preventing ultrastructural kidney damage [11]. In a recent severe porcine hemorrhage study, resuscitation with fresh whole blood resulted in 90\% 72-hour survival compared with $27 \%$ survival in animals receiving standard of care resuscitation fluids (lactated Ringer's, Hextend, 5\% NaCl, 7.5\% NaCl-6\% Dextran-70 [HSD]) [12]. This was a non-coagulopathic model of controlled hemorrhage (53\% bled in 5 minutes), indicating the metabolic benefits of fresh whole blood as a resuscitation solution.

Fresh whole blood transfusion is not without risk. The emergency conditions of the battlefield permit little time to test the blood for infection prior to administration. Aliquots from each unit of transfused fresh whole blood are sent back to the United States for testing. Over 1700 units of fresh whole blood have been transfused in the Iraqi operating room. In the aliquots of this blood, there were two positive confirmatory tests for hepatitis $C(1: 1000)$ (RIBA), and one each indeterminate test (Western blot) of human immunodeficiency virus (HIV) and human Tcell lymphocytic virus (HTLV). Compared to the extremely low incidence of transmission of these diseases in component therapy $(1: 493,000 \mathrm{HIV}, 1: 641,000 \mathrm{HTLV}$, 
$1: 103,000$ hepatitis C, $1: 63,000$ hepatitis B) [13], the risk of infectious disease transmission is still very low, but the surgeon must weigh the risk of infection against the risk of poor outcome if fresh whole blood is not received.

Rapid enzyme-linked immunosorbent assay (ELISA) for disease can be accomplished in two hours. ELISA was successfully utilized at one of the large combat support hospitals and has since seen more widespread use [3]. Without ELISA fresh whole blood must be administered sparingly and only under extreme circum-

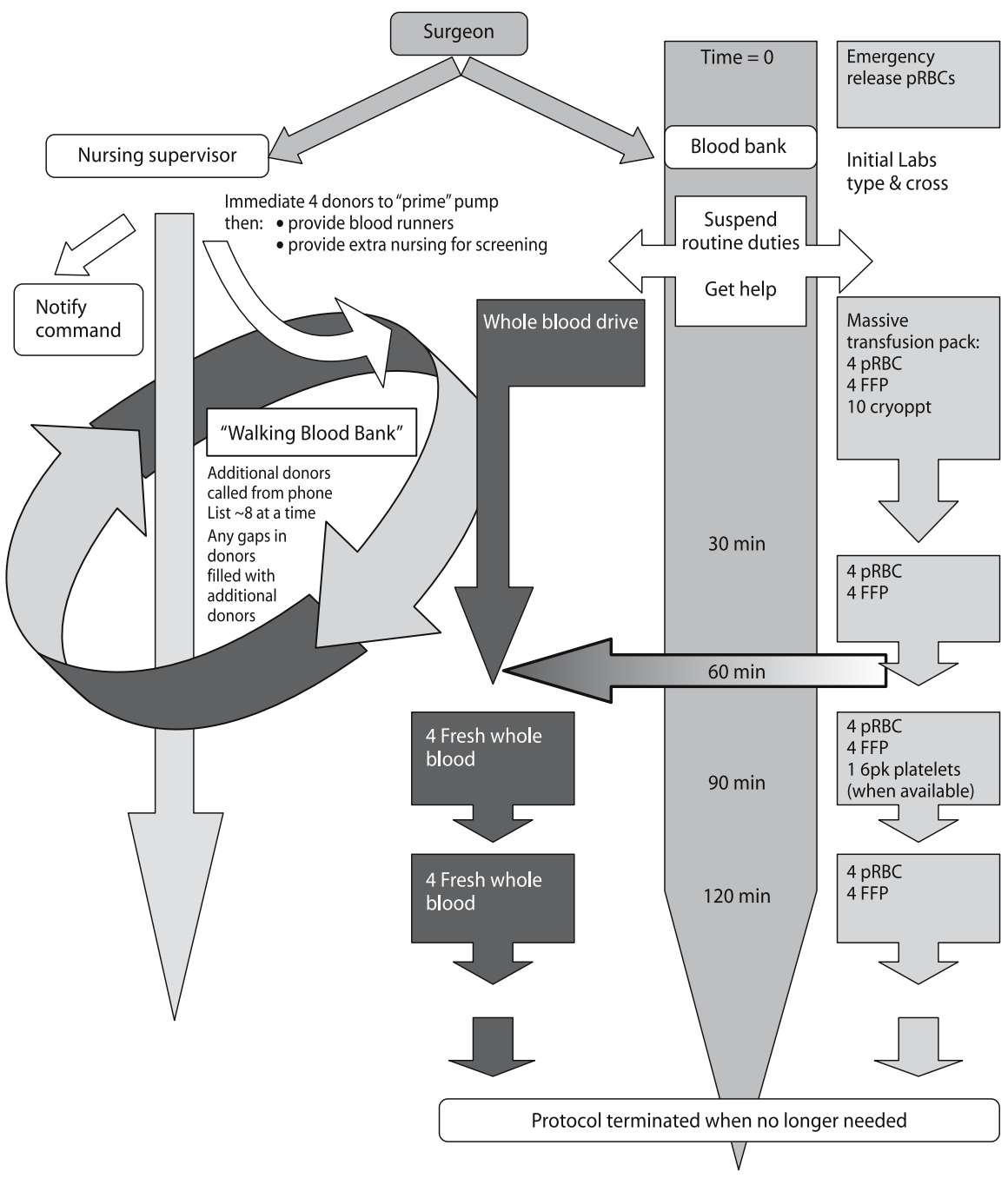

Fig. 1. This flow diagram is the massive transfusion/fresh whole blood transfusion protocol employed by the $31^{\text {st }}$ Combat Support Hospital, while serving in Baghdad, Iraq. This is a two-pronged approach where patients are identified early and the mechanism for obtaining fresh whole blood initiated. Patients receive emergency release packed red blood cells (pRBCs) and when available are transitioned to fresh whole blood. This transition time coincides with the point where platelet transfusion would likely be indicated. FFP: fresh frozen plasma; cryoppt: cryoprecipitate. From [3] with permission 
stances and protocols have been established that delineate the clinical guidelines for determining who should receive fresh whole blood. Figure 1 illustrates one such protocol employed at a combat support hospital [3]. Fresh whole blood was requested when large quantities of blood were required in order to save life and when the onset of coagulopathy and the need for platelets was imminent. This protocol illustrates a two-pronged approach in the event a massive transfusion is required. First, uncrossmatched, Type $O$ packed cells are made available as emergency-release blood and the 'massive transfusion' protocol is initiated. At the same time, since there are usually no platelets and nominal FFP and cryoprecipitate available on the battlefield, the 'walking blood bank' of predetermined donors is activated so that required coagulation factors and components can be provided in the form of fresh whole blood. The first unit of fresh whole blood usually becomes available in about 120 minutes and the surgical team transitions to fresh whole blood as an adjunct to the massive transfusion protocol [3]. Many civilian hospitals utilize this template of a 'massive transfusion protocol' where, upon activation, predetermined quantities of packed RBCs, FFP, platelets and cryoprecipitate are sent at scheduled time intervals. The main difference is the effort to obtain fresh whole blood and the transition to whole blood transfusion.

\section{Itored Packed Red Blood Cells}

Following a landmark study published in the New England Journal of Medicine, the recommendations for red blood cell transfusion when serum hemoglobin levels are less than $10 \mathrm{mg} / \mathrm{dl}$ was changed to hemoglobin levels of $7 \mathrm{mg} / \mathrm{dl}$ in patients with signs of systemic compromise [14]. This randomized controlled trial compared a practice of liberal transfusion (keeping the hematocrit $>30 \%$ ) with a practice of restrictive transfusion (keeping the hematocrit between $21-27 \%$ and only transfusing if the hematocrit fell below 21\%). There was no difference in overall mortality. However, less ill patients (APACHE <20) and younger patients (age < 55) showed a statistically significantly improved survival with the use of a restrictive transfusion. Of clinical significance, the liberal practice group showed more cardiac complications and the restrictive practice population had lower hospital mortality and a lower adjusted multi-organ dysfunction score.

A growing body of literature underscores the potential risks of stored RBC transfusion. A retrospective study assessed over 15,000 patients for the independent predictors of mortality, intensive care unit (ICU) admission, ICU length of stay, and hospital length of stay [15]. After adjustment for injury severity, blood transfusion was shown to be an independent predictor of mortality, ICU admission, and hospital length of stay. Patients who underwent blood transfusion were nearly three times more likely to die and greater than three times more likely to be admitted to the ICU.

Coagulopathy can be caused by the dilution of coagulation factors by packed RBCs which do not contain any factors. Patients who undergo a massive transfusion (more than 10 units of packed RBCs or receipt of twice the normal blood volume) have prolonged prothrombin time, thrombocytopenia and decreased levels of fibrinogen. Seventy percent of those who receive more than 20 units of red blood cells become coagulopathic, with thrombocytopenia being the most common abnormality [16]. A single unit of packed RBCs is approximately $335 \mathrm{ml}$ with a hema- 
tocrit of $55 \%$. A single unit of platelets is $50 \mathrm{ml}$ and contains $5.5 \times 10^{10} / 1$ platelets. A single unit of plasma is about $275 \mathrm{ml}$ with only $80 \%$ coagulation activity of fresh whole blood. When all of these components are combined, the result is a fluid with a hematocrit of $29 \%, 8.8 \times 10^{7} / 1$ platelets and coagulation activity that is $65 \%$ of normal [17]. Even when RBCs are administered at a high one-to-one ratio with FFP, dilution of coagulation factors and platelets and iatrogenic anemia is unavoidable.

It is well known that the trauma related to surgery results in the activation of the immune system. Levels of inflammatory cytokines are elevated to a greater extent in patients with severe blood loss when compared to patients with isolated trauma. Additionally, the degree of elevation of pro-inflammatory cytokines correlates with severity of injury $[18,19]$. When blood transfusion, which is inherently pro-inflammatory, is combined with the inflammatory response associated with trauma, the result is an exacerbated acute phase response [20]. This exacerbated inflammatory response is associated with increased infectious complications and increased mortality [19]. A biphasic inflammatory response begins, with initial proinflammation followed by immunosupression which begins at about 24 hours [18]. It is during the subsequent phase of immunosuppression that the patient is most susceptible to infection. Historically, this immunosuppressive effect was actually used to the advantage of the patient. Prior to the use of cyclosporine, blood transfusion was used in transplant patients as an immunosuppressant, improving outcomes [21].

Several studies further illustrate the disruption of the immune system by RBC transfusion. A prospective trial demonstrated a significantly increased rate of nosocomial infection of $15.4 \%$ in 412 patients who received transfusions compared to a $2.9 \%$ rate in 1,301 control patients who did not receive transfusions [22]. There was a dose-related response between the number of units of packed RBCs received and the risk of developing a nosocomial infection. Additionally, patients who received transfusions showed increased mortality, length of ICU stay, and hospital stay $(\mathrm{p}<0.05)$. This single-center prospective trial corroborated the observations of several basic science studies. Detrimental immunomodulation is thought to occur following increased production of both serum and tissue tumor necrosis factor (TNF)- $\alpha$, interleukin (IL)- $1 \beta$, IL-6, IL- 8 , interacting with soluble cytokine receptors [23]. Fransen et al. showed that surgical patients who received packed RBCs compared to those who did not had increased levels of IL- $6(\mathrm{p}<0.01)$ and bactericidal permeability increasing protein $(\mathrm{BPI})(\mathrm{p}<0.05)$, which is a marker of neutrophil activation [20]. In addition to these serum markers of inflammation, patients who received transfusions had increased ventilator time $(42 \pm 12$ vs. $22 \pm 2 \mathrm{~h}, \mathrm{p}<0.025)$ and increased ICU days ( $45 \pm 6$ vs. $89 \pm 21, \mathrm{p}<0.025)$ [20]. Transfusion of blood components has also been associated with impaired natural killer cell function and decreased helper-suppressor cell ratios as well as effects on B lymphocytes [24]. Again, there appeared to be a linear relationship between the amount of blood transfused and the degree of impairment [24].

Approximately twenty-four hours is required for transfused packed RBCs to recover their full ability to deliver oxygen, but the precise mechanism of this immunomodulation is a mystery. One theory is that immune system derangement is the result of the storage age of blood. Gastric mucosal $\mathrm{pH}$ can be used as an indicator of oxygenation of the gastric mucosa or the organs of splanchnic distribution and studies have shown that direct measurements of gastric mucosal $\mathrm{pH}$ are related to the age of the blood transfused to critically ill patients $[25,26]$. Two mechanisms 
that have been proposed to account for the inability of old transfused RBCs to improve systemic oxygen consumption include a left shift in the oxyhemoglobin dissociation curve due to 2,3-diphosphoglycerate (DPG) depletion with storage, and loss of the RBC compliance and the ability to deform, impeding delivery of oxygen to the microcirculation [27]. Another emerging theory speculates that due to the high affinity of nitric oxide (NO) with free hemoglobin, cell-free ferrous hemoglobin in the plasma is oxidized to methemoglobin and nitrates that rapidly destroy NO $[28,29]$. The decreased availability of NO results in regional and systemic vasoconstriction.

The exact mechanisms are uncertain, but it is becoming clearer that the administration of stored RBCs is a clinical decision that should not be taken lightly. The negative repercussions of packed $\mathrm{RBC}$ transfusion may become evident in trauma patients, who present in a state of acute inflammation derived from the trauma sustained. The literature supports the theory that the transfusion of packed RBCs can actually result in a deranged inflammatory response and that this may be related to the storage age of the blood. The transfusion of packed RBCs remains the standard of care for restoring RBC concentration and circulating oxygen content during ongoing hemorrhage, but it is not a solution that is devoid of risk.

\section{Hemoglobin-Based Oxygen Carriers}

In severe hemorrhage, oxygen delivery to tissues must be restored. Hence, the rationale for hemoglobin-based oxygen carriers is the ability to deliver oxygen to vital organs via plasma components. But the development of hemoglobin-based oxygen carriers to replace packed RBCs has led a rocky course over the past twenty-five years. Several variants of hemoglobin-based oxygen carriers attempted in the past were either abandoned by the developing company or discontinued in phase III trials out of concern for patient safety [30]. Concerns about hemoglobinbased oxygen carriers are their effects on vasoactivity resulting from binding of NO by hemoglobin and subsequent hypertension. Currently, however, in the United States a phase III trial of pyridoxalated hemoglobin (Polyheme, Northfield Laboratories, Evanston, IL.) is 50\% completed. This multicenter pre-hospital trial is examining the administration of hemoglobin based oxygen carriers to trauma patients in severe hemorrhagic shock. Hopefully, upon completion of this study, hemoglobin based oxygen carriers will be added to the armamentarium of the caregiver.

An effective and safe hemoglobin-based oxygen carrier appeals to both the civilian and military communities. Polyheme can be stored for 72 hours at room temperature, virtually eliminates any risk of disease transmission, does not require type and cross-matching and provides a fluid which can enhance oxygen delivery [31]. It does not, however, have any restorative effect on coagulation. For military use, Polyheme will require availability at room temperature extended beyond 72 hours. Whether it will be carried in the rucksack of a medic for a limited time or in a civilian ambulance, this potentially lifesaving product deserves further exploration. 


\section{Fresh Frozen Plasma}

Plasma contains various components including stable coagulation factors, fibrinogen and albumin. Lyophilized plasma was the principle resuscitation fluid during both World War II and the Korean War due to its long shelf life without refrigeration. At the end of World War II and the Korean War, the discovery of infectious diseases as the result of plasma obtained from multiple donors, i.e., pooled plasma, halted this practice. Consequently, the indications for plasma transfusion today no longer include that of a volume expander. FFP is indicated for the correction of coagulopathy associated with factor deficiency (II, VII, IX, X), hemorrhage, reversal of warfarin effect, and antithrombin deficiency.

During massive transfusion of packed RBCs in a patient with exsanguinating hemorrhage, the onset of coagulopathy is usually described as the result of dilution and consumption of coagulation factors, acidosis and hypothermia. The incidence of coagulopathy increases with injury severity. Twenty-one percent of patients with an injury severity score (ISS) of 15-29 are coagulopathic, 41\% with an ISS of $30-$ $44,59 \%$ with ISS of $45-59$, and $79 \%$ of those with an ISS of $60-75$ [32]. However, importantly, the most seriously injured patients are coagulopathic upon admission, unrelated to dilution. In a large study of 1,088 trauma patients, $24.4 \%$ were coagulopathic on admission [32]. As a general rule, hemostasis is possible as long as coagulation factor activity is kept at 20 to $30 \%$ of normal and fibrinogen levels are at least $100 \mathrm{mg} / \mathrm{dl}$. FFP contains plasma clotting factors and fibrinogen; in addition, administration will increase intravascular volume.

Because the incidence of coagulopathy increases with severity of injury, many institutions include the transfusion of single donor FFP in their massive transfusion protocols. Often, the first application of blood products contains six units of type $\mathrm{O}$ RBCs and four units of $\mathrm{AB}$ negative FFP. Subsequent applications include six units of type specific RBCs and FFP, and a six-pack of platelets with a unit of cryoprecipitate in every other application. Cryoprecipitate provides fibrinogen and some clotting factors which become depleted in a massive transfusion. Volume expansion inherently occurs with the administration of these products and is also augmented with either crystalloid or colloid solutions. The purpose of this protocol is to restore circulating RBCs, volume, clotting factors, and clotting substrate and to sustain the patient while the surgeon stops the source of bleeding.

The onset of coagulopathy in the hemorrhaging patient can be rapid, and the time required to obtain plasma can further propel the patient along the 'bloody vicious cycle' of coagulopathy, acidosis, and hypothermia. Therefore, to prevent a dangerous situation the caregiver must be ready to transfuse blood products based on immediate clinical evaluation rather than delayed laboratory assessment. The current laboratory guidelines for the transfusion of FFP are prothrombin time (PT) more than 1.5 times greater than normal, activated partial thromboplastin time (aPTT) ratio more than 1.5 times greater than normal, fibrinogen less than $0.8 \mathrm{~g} / \mathrm{l}$, and coagulation factor levels $30 \%$ of normal [17]. Some authors advocate early FFP transfusion at a 1:1 ratio with packed RBCs after hemorrhage of one blood volume, or PT and aPTT greater than 1.5 times normal with ongoing hemorrhage [33]. Even with this aggressive practice of plasma transfusion, hemodilution is unavoidable. As stated earlier, when standard blood products are administered in a 1:1 ratio the resulting fluid has a hematocrit of $29 \%, 8.8 \times 10^{7} / 1$ platelets, and coagulation activity that is $65 \%$ of normal [17]. The primary risks associated with fresh frozen plasma transfusion are infection, transfusion-related acute lung injury (TRALI), acute aller- 
gic and anaphylactic reactions, hemolysis due to anti-A and anti-B, and fluid overload [34]. Of these, the most common serious complication of FFP transfusion is TRALI, thought to occur in a graft versus host mechanism as donor antibodies react with host leukocytes [35]. The rate of infectious disease transmission resulting from FFP administration is similar to that of packed RBCs.

\section{Platelets}

It is important that clinicians take into consideration the etiology of thrombocytopenia, platelet dysfunction, risk of bleeding, planned invasive procedures, and the presence of concomitant disorders when deciding whether or not to transfuse platelets. Patients with severe sepsis, regardless of the presence of hemorrhage, usually receive a transfusion of platelets if their platelet count falls below $5000 / \mathrm{mm}^{3}$. Platelet transfusion should be considered if the platelet count is between 5000-30,000/ $\mathrm{mm}^{3}$, and there are signs of bleeding. If the patient requires surgery or other invasive procedures it is recommended that the platelet count be maintained above $50,000 / \mathrm{mm}^{3}$. The conundrum presented by these classic recommendations is the highly variable relationship between platelet counts and platelet efficacy.

Platelets pose significant logistical problems. Since the development of platelet aphaeresis technology, it has been well known that platelets stored in the cold have poor recovery and survival in vivo. They cannot be refrigerated even for short periods of time, thus platelets are stored at $22^{\circ} \mathrm{C}$. Studies of chilled platelets have shown that this irreversibly alters their morphology as well as the expression of the $\mathrm{GPIb} a$ receptor on the platelet, causing rapid clearance of the transfused platelet from the circulation [36]. The changes which occur in the structure and function of stored platelets are known as the 'platelet storage lesion' and are poorly understood. There are few data regarding the function of platelets in the bleeding patient. After room temperature storage of up to 5 days, the risk of bacterial contamination becomes significant. The rate of septic reaction to platelet transfusion in the United States is between $1: 10,000$ and $1: 20,000$ [37]. The short shelf-life of platelets combined with an uncertain demand for platelets results in wastage rates as high as $50 \%$.

Significant efforts are being made in the development of novel platelet products and platelet substitutes. Lyophilized platelets are one such product, initially investigated nearly fifty years ago and recently resurfaced [38]. Lyophilized platelets have shown encouraging preclinical results in animal models [39]. A second product, Synthocyte $^{\mathrm{TM}}$ (Profibrix, Inc., The Netherlands), is a fibrinogen-coated albumin microsphere that has shown a reduction in surgical bleeding in a thrombocytopenic animal model [40]. The mechanism of action of Synthocytes ${ }^{\mathrm{TM}}$ is thought to be due to the cross-linking of fibrinogen which has been directly imbedded into the surface of the microcapsules [41]. Other areas of platelet product development include liposome-based hemostatic agents, thromboerythrocytes, and platelet-derived microparticles. 


\section{Fibrinogen}

One of the end products of the coagulation system is the production of thrombin which stimulates the sequential proteolytic cleavage of fibrinogen to release fibrinopeptides $\mathrm{A}$ and $\mathrm{B}$. The fibrin monomers that result from this process spontaneously polymerize to form an insoluble matrix. This matrix is stabilized by factor XIIIa, which is another product of thrombin generation. Fibrinogen, or factor I, is essential for hemostasis and it is recommended that fibrinogen be administered in the form of cryoprecipitate once fibrinogen levels fall below $100 \mathrm{mg} / \mathrm{dl}$. This number applies to patients with non-surgical bleeding and not necessarily to those with active blood loss.

Cryoprecipitate derived from a unit of whole blood contains $10-20 \mathrm{ml}$ of fluid per unit, providing up to $150-250 \mathrm{mg}$ of fibrinogen, 80-100 units of factor VIII, and 50-60 mg of fibronectin and in a non-bleeding $70 \mathrm{~kg}$ patient one pool of cryoprecipitate will increase the fibrinogen levels $45 \mathrm{mg} / \mathrm{dl}$. Cryoprecipitate does not contain all of the necessary coagulation factors and should not be used in place of FFP.

Fibrinogen substitutes are being actively investigated for fibrinogen supplementation. Recombinant fibrinogen, rhFIB, (Pharming Group, Denmark) is currently under investigation by the United States Army as an adjunct for hemostasis. Another group has shown that fibrinogen derived from salmon activates human platelets and may provide a hemostatic function [42]. A third product, Haemocomplet$\tan$ (Aventis Behring GmbH, Marburg, Germany), is a lyophilized human fibrinogen concentrate that has been shown to correct thromboelastograph abnormalities and improve mortality as seen in rat models of sepsis-induced disseminated intravascular coagulation (DIC) [43]. Fibrinogen substitutes would allow more rapid correction of fibrinogen dilution than cryoprecipitate.

\section{Recombinant Factor VIla}

Over the past several years recombinant factor VIIa (rFVIIa) (Novoseven, Novonordisk, Malov, Denmark) has emerged as a promising therapy for treatment of coagulopathy associated with trauma and massive transfusion. This emergence began as observational experiences [44], translated into pre-clinical studies in animal models $[45,46]$, and progressed to large, multicenter, randomized and controlled studies which clearly show the safety and efficacy of rFVIIa in severely injured trauma patients. Phase II trials will soon advance to larger phase III studies and rFVIIa holds promise as an adjunct to hemostasis in patients who receive massive transfusions for both blunt and penetrating trauma.

Used as a surgical adjunct rFVIIa reduces blood loss. In a randomized, doubleblinded prospective study, surgical patients who underwent a retropubic prostatectomy were given $20 \mu \mathrm{g} / \mathrm{kg}$ rFVIIa, $40 \mu \mathrm{g} / \mathrm{kg}$ rFVIIa or placebo prior to surgery. The higher dose group showed significant decreases in blood loss $(\mathrm{p}<0.01)$ and surgical time $(\mathrm{p}<0.05)$ and elimination of packed RBC transfusion requirements [47]. On the other hand, studies regarding the proper administration of rFVIIa have elucidated that rFVIIa is ineffective as a 'last resort' [48]. We recommend that the administration of rFVIIa follow a clinical protocol according to set guidelines for dose and other parameters in order to optimize success. One early guideline published 
by Dutton et al. outlines the use of clinical "gatekeepers" who are available to assess the patient and clinical circumstances surrounding the administration of rFVIIa [49]. Criteria include evidence of ongoing active hemorrhage, with clinical evidence of coagulopathy, and ongoing utilization of conventional transfusion and hemostatic therapy which must be judged unlikely to succeed. Patients must have received at minimum 10 units of RBCs, eight units of FFP, one unit of platelets, with continued derangement of coagulation studies and ongoing hemorrhage. This approach reserves $\mathrm{rFVII}$ for the most critically injured.

With its safety demonstrated in a large randomized clinical trial, most clinicians are utilizing rFVIIa earlier in an attempt to prevent massive bleeding and coagulopathy rather than treating them after the fact. In a protocol in May 2004 from the Trauma Consultant to the Surgeon General of the United States, this change from reacting to coagulopathy to actively preventing coagulopathy with rFVIIa was made manifest. It is recommended that combat surgeons "consider rFVIIa administration for use in patients that require damage control procedures, have coagulopathic bleeding, or difficult to control bleeding associated with hypothermia or significant operative bleeding." It is further recommended that the surgeon consider administering two units of fresh whole blood prior to rFVIIa to ensure the presence of functional platelets and coagulation factors.

A significant drawback to the administration of rFVIIa is cost. On average, an $80 \mathrm{~kg}$ man receiving a $120 \mu \mathrm{g} / \mathrm{kg}$ dose, costs about $\$ 8,000$. Though this cost appears exorbitant, most studies demonstrate a reduction in blood product requirements, which in turn should translate to lower costs and prevention of subsequent infection and deleterious inflammatory response. Efforts are also being taken to decrease cost by making rFVIIa more efficient. In a rat model of hemophilia, three 'super analogs' of rFVIIa were compared with rFVIIa [50]. The rFVIIa groups received $1,3,6$ or $10 \mathrm{mg} / \mathrm{kg}$, and the super-analog groups 1 or $3 \mathrm{mg} / \mathrm{kg}$. Two of the $3 \mathrm{mg} / \mathrm{kg}$ groups of the super-analogs and the $10 \mathrm{mg} / \mathrm{kg}$ group of rFVIIa showed significant improvement in bleeding times $(\mathrm{p}<0.001)$ when compared to the hemophilia control group. Average blood loss was reduced in the 3, 6 and $10 \mathrm{mg} / \mathrm{kg}$ groups of rFVIIa $(\mathrm{p}<0.05)$ and in all groups of the super-analog, including the $1 \mathrm{mg} / \mathrm{kg}$ groups $(\mathrm{p}<0.05)$. A dose response was seen between the 1 and $3 \mathrm{mg} / \mathrm{kg}$ groups of all three super-analogs, with decreased blood volumes in the higher dose super analog groups $(p<0.001)$ [50]. With further research and the development of rFVIIa analogs with greater potency, we hope to see the cost associated with this drug decrease while effectiveness increases.

\section{Where are we Heading?}

The potential hematologic and physiologic benefits of fresh whole blood as a hemostatic and resuscitative fluid are clear. This straightforward approach is akin to the damage control procedures so widely accepted in trauma surgery, although it is temporized by inherent logistical problems and the small but real threat of infectious diseases. What is needed is a fluid which contains the oxygen-carrying capability as well as the volume and hemostatic qualities provided by fresh whole blood. For hemostatic resuscitation on the battlefield we also envision a fluid that is stable at room temperature and has a long shelf life. Additionally, a fluid that can transport oxygen, possibly by a hemoglobin-based oxygen carrier could serve 
as a volume expander and contain active clotting factors and platelets, like those found in freeze-dried plasma, platelets, fibrinogen and recombinant factor VIIa. Being fielded forward to the point of injury, this fluid could prevent the onset of coagulopathy and decrease blood product transfusion requirements, all in hope of improving survival. Fascinating and promising products on the horizon may make hemostatic resuscitation a possibility.

\section{Conclusion}

Component therapy is useful for the majority of patients when blood requirements are minimal and there is no associated coagulopathy. Of concern are requirements for massive transfusion and resuscitation that absorb resources and create a shortfall for patients whose injuries are less severe. Additionally, the conventional massive transfusion model of packed RBCs, plasma and platelets actually further dilutes the patient compared to the blood he or she has lost and thus is not the ideal fluid for patients who require this massive transfusion of products. Fresh whole blood has three vital properties: oxygen carrying capacity, volume, and hemostatic effect. In the austere environment of combat the practice of fresh whole blood transfusion has proven beneficial to patients who are coagulopathic and require massive transfusion. Appropriate use following established guidelines can be beneficial and may even be superior to packed RBCs. A fluid containing the vital properties of fresh whole blood would serve as a bridge to allow a patient to be resuscitated without initiating the 'bloody cycle of death' that is seen all too often in our current paradigm of massive resuscitation.

\section{References}

1. Grosso SM, Keenan JO (2000) Whole blood transfusion for exsanguinating coagulopathy in a US field surgical hospital in postwar Kosovo. J Trauma 49:145-148

2. Mabry RL, Holcomb JB, Baker AM, et al (2000) United States Army Rangers in Somalia: an analysis of combat casualties on an urban battlefield. J Trauma 49:515-528

3. Repine TB, Perkins J, Kauvar D, Blackborne L (2006) The use of fresh whole blood in massive transfusion. J Trauma (in press)

4. Pearce FJ, Lyons WS (1999) Logistics of parenteral fluids in battlefield resuscitation. Mil Med 164:653-655

5. Kendrick DB (1964) Blood Program in World War II. Office of the Surgeon General, Washington DC, pp 781

6. Mohr R, Martinowitz U, Lavee J, et al (1988) The hemostatic effect of transfusing fresh whole blood versus platelet concentrates after cardiac operations. J Thorac Cardiovasc Surg 96:530-534

7. Manno CS, Hedberg KW, Kim HC, et al (1991) Comparison of the hemostatic effects of fresh whole blood, stored whole blood, and components after open heart surgery in children. Blood 77:930-936

8. Barbee RW, Kline JA, Watts JA (1999) A comparison of resuscitation with packed red blood cells and whole blood following hemorrhagic shock in canines. Shock 12:449-453

9. Curtis SE, Cain SM (1992) Regional and systemic oxygen delivery/uptake relations and lactate flux in hyperdynamic, endotoxin-treated dogs. Am Rev Respir Dis 145:348-354

10. Turranoglu S, Kaya S, Kararmaz A, Turhanoglu AD (2001) Lung perfusion in hemorrhagic shock of rats: the effects of resuscitation with whole blood, saline or Hes 6\%. Tohoku J Exp Med 195:245-251 
11. Onen A, Cigdem MK, Deveci E, et al (2003) Effects of whole blood, crystalloid, and colloid resuscitation of hemorrhagic shock on renal damage in rats: an ultrastructural study. J Pediatr Surg 38:1642-1649

12. Sondeen JL, Dubick MA, Prince DM, Holcomb JB (2005) Fresh whole blood is the best hypotensive resuscitative fluid in a severe hemorrhage pig model. Circulation (abst, in press)

13. Schreiber GB, Busch MP, Kleinman SH, Korelitz JJ (1996) The risk of transfusion-transmitted viral infections. The Retrovirus Epidemiology Donor Study. N Engl J Med 334:16851690

14. Hebert PC, Wells G, Blajchman MA, et al (1999) A multicenter, randomized, controlled clinical trial of transfusion requirements in critical care. Transfusion Requirements in Critical Care Investigators, Canadian Critical Care Trials Group. N Engl J Med 340:409-417

15. Malone DL, Dunne J, Tracy JK, et al (2003) Blood transfusion, independent of shock severity, is associated with worse outcome in trauma. J Trauma 54:898-905

16. Faringer PD, Mullins RJ, Johnson RL, Trunkey DD (1993) Blood component supplementation during massive transfusion of AS-1 red cells in trauma patients. J Trauma 34:481-485

17. Armand R, Hess JR (2003) Treating coagulopathy in trauma patients. Transfus Med Rev $17: 223-231$

18. Angele MK, Faist E (2002) Clinical review: immunodepression in the surgical patient and increased susceptibility to infection. Crit Care 6:298-305

19. Roumen RM, Hendriks T, van der Ven-Jongekrijg J, et al (1993) Cytokine patterns in patients after major vascular surgery, hemorrhagic shock, and severe blunt trauma. Relation with subsequent adult respiratory distress syndrome and multiple organ failure. Ann Surg 218:769-776

20. Fransen E, Maessen J, Dentener M, Senden N, Buurman W (1999) Impact of blood transfusions on inflammatory mediator release in patients undergoing cardiac surgery. Chest 116:1233-1239

21. Pfaff WW, Fennell RS, Howard RJ, Ireland JF, Scornik JC (1984) Planned random donor blood transfusion in preparation for transplantation. Sensitization and graft survival. Transplantation 38:701-703

22. Taylor RW, Manganaro L, O’Brien J, et al (2002) Impact of allogenic packed red blood cell transfusion on nosocomial infection rates in the critically ill patient. Crit Care Med 30: 2249-2254

23. Bengtsson A, Redl H, Schlag G, Mollnes TE, Hogasen K (1996) Effects on complement activation and cytokine (TNF-alpha and IL-8) release of infusion of anti-TNF-antibodies or a xanthine derivative (HWA 138) in septic baboons. Acta Anaesthesiol Scand 40:244-249

24. Landers DF, Dullye KK (1999) Vascular access and fluid resuscitation in trauma. Anesth Clin N Am 17:125-140

25. Marik PE, Sibbald WJ (1993) Effect of stored-blood transfusion on oxygen delivery in patients with sepsis. JAMA 269:3024-3029

26. Silverman HJ, Tuma P (1992) Gastric tonometry in patients with sepsis. Effects of dobutamine infusions and packed red blood cell transfusions. Chest 102:184-188

27. Morisaki H, Sibbald WJ (2004) Tissue oxygen delivery and the microcirculation. Crit Care Clin 20:213-223

28. Reiter CD, Wang X, Tanus-Santos JE, et al (2002) Cell-free hemoglobin limits nitric oxide bioavailability in sickle-cell disease. Nat Med 8:1383-1389

29. Schechter AN, Gladwin MT (2003) Hemoglobin and the paracrine and endocrine functions of nitric oxide. N Engl J Med 348:1483-1485

30. Sloan EP (2003) The clinical trials of diaspirin cross-linked hemoglobin (DCLHb) in severe traumatic hemorrhagic shock: the tale of two continents. Intensive Care Med 29:347-349

31. Moore EE, Johnson JL, Cheng AM, Masuno T, Banerjee A (2005) Insights from studies of blood substitutes in trauma. Shock 24:197-205

32. Brohi K, Singh J, Heron M, Coats T (2003) Acute traumatic coagulopathy. J Trauma 54: $1127-1130$

33. Ho AM, Karmakar MK, Dion PW (2005) Are we giving enough coagulation factors during major trauma resuscitation? Am J Surg 190:479-484

34. Maclennan S (2006) Risks of fresh frozen plasma (FFP) and platelets. J Trauma (in press) 
35. Popovsky MA, Moore SB (1985) Diagnostic and pathogenetic considerations in transfusionrelated acute lung injury. Transfusion 25:573-577

36. Hoffmeister KM, Felbinger TW, Falet H, et al (2003) The clearance mechanism of chilled blood platelets. Cell 112:87-97

37. Engelfriet CP, Reesink HW, Blajchman MA, et al (2000) Bacterial contamination of blood components. Vox Sang 78:59-67

38. Blajchman M (2001) Novel platelet products, substitutes and alternatives. Transfus Clin Biol 8:267-271

39. Read MS, Reddick RL, Bode AP, et al (1995) Preservation of hemostatic and structural properties of rehydrated lyophilised platelets: potential for long-term storage of dried platelets for transfusion. Proc Natl Acad Sci USA 36:536-542

40. Levi M, Friederich PW, Middleton S, et al (1999) Fibrinogen-coated albumin microcapsules reduce bleeding in severely thrombocytopenic rabbits. Nat Med 5:107-111

41. Davies AR, Judge HM, May JA, Glenn JR, Heptinstall S (2002) Interactions of platelets with Synthocytes, a novel platelet substitute. Platelets 13:197-205

42. Manseth E, Skjervold PO, Fjaera SO, et al (2004) Purification and characterization of Atlantic salmon (Salmo salar) fibrinogen. Comp Biochem Physiol B Biochem Mol Biol 138:169174

43. Kaspereit F, Doerr B, Dickneite G (2004) The effect of fibrinogen concentrate administration on coagulation abnormalities in a rat sepsis model. Blood Coagul Fibrinolysis 15:39-43

44. Kenet G, Walden R, Eldad A, Martinowitz U (1999) Treatment of traumatic bleeding with recombinant factor VIIa. Lancet 354:1879

45. Schreiber MA, Holcomb JB, Hedner U, et al (2002) The effect of recombinant factor VIIa on coagulopathic pigs with grade $\mathrm{V}$ liver injuries. J Trauma 53:252-257

46. Sondeen JL, Pusateri AE, Hedner U, Yantis LD, Holcomb JB (2004) Recombinant factor VIIa increases the pressure at which rebleeding occurs in porcine uncontrolled aortic hemorrhage model. Shock 22:163-168

47. Friederich PW, Henny CP, Messelink EJ, et al (2003) Effect of recombinant activated factor VII on perioperative blood loss in patients undergoing retropubic prostatectomy: a doubleblind placebo-controlled randomised trial. Lancet 361:201-205

48. Clark AD, Gordon WC, Walker ID, Tait RC (2004) 'Last-ditch' use of recombinant factor VIIa in patients with massive haemorrhage is ineffective. Vox Sang 86:120-124

49. Dutton RP, McCunn M, Hyder M, et al (2004) Factor VIIa for correction of traumatic coagulopathy. J Trauma 57:709-718

50. Tranholm M, Kristensen K, Kristensen AT, et al (2003) Improved hemostasis with superactive analogs of factor VIIa in a mouse model of hemophilia A. Blood 102:3615-3620 